\title{
Imagen de la enfermería a través de la prensa escrita ¿necesitamos visibilizar los cuidados enfermeros?
}

\author{
Nursing's image in the press. \\ Do we need greater recognition for nursing care? \\ Imagem da enfermagem por meio da imprensa. \\ Será que precisamos visibilizar os cuidados enfermeiros?
}

Selene Sánchez-Gras

Graduada en Enfermería por la Universidad de Alicante.

Cómo citar este artículo en edición digital: Sánchez-Gras, S. (2017). Imagen de la enfermería a través de la prensa escrita ¿necesitamos visibilizar los cuidados enfermeros? Cultura de los Cuidados (Edición digital), 21(49).

Recuperado de http://dx.doi.org/10.14198/cuid.2017.49.08

Correspondencia: Remitirse al correo electrónico.

Correo electrónico:ssg48@alu.ua.es

Recibido: 11/03/2017; Aceptado: 11/06/2017

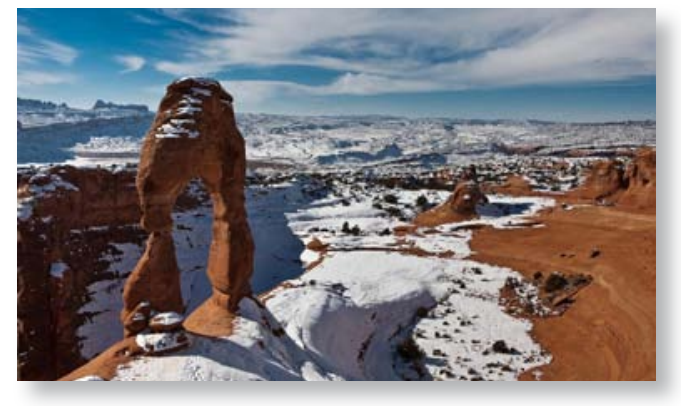

ABSTRACT

Introduction: several studies show that the public image of nurses and their professional image are not related. The social image of a profession is very conditioned by the way it is represented in the media. Therefore, the aim of the study was to present a critical and exhaustive analysis of the treatment received by the profession and the professional nursing in the daily press.

Material and Methods: in the first phase of the study has been conducted a literature reviews to know the status of the matter. Subsequently, is proposed a qualitative and analytical study about the sources published in regional and national newspapers in which news apperars nursing.
Results: a total of 235 news were selected and analyzed. After analysis of their content, eight categories that help explain the reflected image of nursing in the press emerged.

Discussion / Conclusions: in general, the image is transmitted in the press is the image of someone with a secondary role associated with another profession, without responsibility, autonomy or ability in decision process. This image must be changed by ourselves.

Keywords: nursing, communications media, mass media, social image, visibility.

\section{RESUMEN}

Introducción: diversos estudios muestran que la imagen pública de las enfermeras no siempre coincide con su imagen profesional. La imagen social de una profesión está muy condicionada por la forma en que es reproducida en los medios de comunicación. Por ello, el objetivo del estudio fue presentar un análisis crítico y exhaustivo del tratamiento que recibe la profesión y el profesional de Enfermería en la prensa escrita.

Material y método: en la primera fase del 
estudio se ha realizado una revisión bibliografía para conocer el estado de la cuestión. Posteriormente, se propone un estudio cualitativo y analítico utilizando como fuentes las noticias publicadas en la prensa escrita regional y nacional en las que aparece la Enfermería.

Resultados: se seleccionaron y analizaron un total de 235 noticias. Tras el análisis de contenido de las mismas, emergieron 8 categorías que ayudan a explicar la imagen que se refleja de la enfermería en la prensa.

Discusión / Conclusiones: en general, la imagen que tansmite la prensa es la imagen de alguien con un papel secundario asociado a otra profesión, sin responsabilidad, autonomía o capacidad en la toma de decisiones. Cambiar la imagen que transmitimos se encuentra en nuestras propias manos.

Palabras clave: enfermería, medios de comunicación, medios de comunicación de masas, imagen social, visibilidad.

\section{RESUMO}

Introdução: Vários estudos mostram que a imagem pública de enfermeiros nem sempre coincidem com a sua imagem profissional. A imagem social de uma profissão é muito condicionada pela forma como ele é reproduzido na mídia. Portanto, o objetivo do estudo foi apresentar uma análise crítica e abrangente do tratamento recebido pela profissão e do profissional de enfermagem na imprensa.

Material e métodos: na primeira fase do estudo foi conduzido revisões de literatura de saber o estado da questão. Posteriormente, um estudo qualitativo e analítico utilizando como fontes publicadas em jornais regionais e nacionais em que notícias aparece Enfermagem é proposto.

Resultados: Foram selecionados e analisados um total de 235 notícias. Após a análise do seu conteúdo, eles emergiram oito categorias que ajudam a explicar a imagem refletida da enfermagem na imprensa.

Discussão / Conclusão: No geral, a imagem através da imprensa transmite é a imagem de alguém com um papel secundário associado a outra profissão, sem responsabilidade, autonomia ou habilidade na tomada de decisões. Você alterar a imagem que transmitimos estamos em nossas próprias mãos.

Palavras chave: enfermagem, meios de comunicação, meios de comunicação de massa, imagem social, visibilidade.

\section{INTRODUCCIÓN}

La Enfermería es una profesión que ha sufrido, durante muchos años, del estereotipo público. Ha estado sujeta a ser vista como una profesión predominantemente femenina, bajo el mandato médico, sin un campo competencial propio y con falta de profesionalidad (Kemmer y Silva, 2007; Errasti-Ibarrondo, Arantzamendi-Solabarrieta y Canga-Armayor, 2012). A pesar de los logros profesionales $\mathrm{y}$ académicos obtenidos durante las últimas décadas, parece ser que no se ha cambiado la percepción que tiene la opinión pública sobre la Enfermería, continuando su imagen plagada de estereotipos (Heirle, 2009; Calvo, 2011). La falta de visibilidad de los cuidados enfermeros se refleja en estudios como los propuestos por Takase, Kershaw y Burt (2002), Ten Hoeve, Jansen y Roodbol (2013) o Martins, García y Correia (2014). En estos estudios queda patente que la imagen pública de las enfermeras no siempre coincide con su imagen profesional; las enfermeras no son representadas como los profesionales autónomos que son y el público no es consciente de la situación académica, científica y profesional actual de la Enfermería. 
Existen varias razones que pueden contribuir a este escaso reconocimiento social que se transforma en la invisibilidad de la profesión y la esencia de la misma, el cuidado. Una de ellas puede ser su comienzo histórico, se encontraba vinculada al género femenino y de origen doméstico, de esta manera, se le confería un carácter obligatorio al cuidado carente de logros (Marchador, 2005). Por otra parte, la actitud de los propios profesionales, según Fernández y Ramos (2013) deberíamos diseñar un plan de comunicación para proyectar nuestra "voz" con un lenguaje claro y sencillo para mantenernos en el mismo canal de comunicación que los pacientes. Además, incluye que necesitamos registrar correctamente nuestro trabajo e incluso investigarlo, así como oponernos a aquellas tareas que no aportan valor a nuestra labor. Otro factor identificado que contribuye a acrecentar la invisibilidad es la presencia de los denominados cuidados invisibles. Se trata de cuidados de carácter más humano, con dificultad para ser cuantificados y escasamente registrados, pero que son aquellos cuidados profesionales que nos identifican y diferencian, como por ejemplo, estrategias comunicativas, estrategias creativas para responder a las necesidades de cuidado o cuidados relacionados con el alivio, el confort o la intimidad (Huércanos, 2010; Huércanos, 2013; Orkaizagirre, 2013; Bonill, 2014).

La imagen social de una profesión está muy condicionada por la forma en que es reproducida a través de los medios de comunicación. Esta forma, es la que contribuye a configurar el concepto que la sociedad posee de tal profesión. (Kalisch, Begeny y Neumann, 2007; Rodríguez, Rodríguez y Azañón, 2008; Heirle, 2009; Calvo, 2011).

Entre los motivos que han contribuido a centrar el tema en este sentido se encuentra el interés por comprender mejor las repercusiones que conlleva la imagen negativa de una profesión y la identificación de estrategias de actuación. Según Errasti-Ibarrondo et al. (2012) si la población tiene una idea errónea o incompleta de lo que es el quehacer enfermero puede desembocar en numerosas consecuencias, como que la población no acuda a ella y se beneficie de sus servicios. Además, podría tener un impacto en la cantidad de personas que la eligen como profesión o afectar a la toma de decisiones de los políticos, que definen el alcance y financiación de los servicios (McAllister, Downer, Hanson y Oprescu, 2014). Por otro lado, la imagen que las enfermeras tienen de sí mismas puede verse afectada, causándoles frustración e incidiendo en el rendimiento del trabajo enfermero (Heirle, 2009; Kalisch et al., 2007). Todo lo anteriormente mencionado, nos lleva a enunciar la pregunta de investigación a la que se dirige este estudio: ¿Cuál es la imagen de la profesión enfermera que se difunde a través de la prensa escrita? Este trabajo partía de la hipótesis de que entre la imagen real de la Enfermería y la que se transmite en los medios de comunicación hay un distanciamiento.

\section{OBJETIVO}

Para responder a la pregunta planteada el objetivo que se propuso fue presentar un análisis crítico y exhaustivo del tratamiento que recibe la profesión y el profesional de Enfermería en la prensa escrita diaria.

\section{MATERIAL Y MÉTODO}

La realización del estudio ha constado de 2 fases. En la primera fase se ha realizado una revisión bibliografía para conocer el estado de la cuestión. Posteriormente, se propone un estudio cualitativo y analítico utilizando como 
fuentes las noticias publicadas en la prensa escrita regional y nacional en las que aparece la Enfermería.

La revisión bibliográfica se llevó a cabo en las bases de datos CUIDEN, Medline (a través de Pubmed), Scielo, CINAHL y se amplió la misma usando el portal de búsqueda Google académico. La restricción de fecha aplicada fue de 15 años, por tanto se incluyen estudios publicados desde el año 2000 hasta mayo de 2015. Los términos de búsqueda empleados fueron: enfermería, enfermeras, medios de comunicación, medios de comunicación de masas e imagen social y sus respectivas correlaciones en inglés. Para la búsqueda en Medline y CINAHL se utilizaron los descriptores disponibles del Medical Subject Headings (MeSH) y para Scielo y CUIDEN los del tesauro de descriptores en Ciencias de la Salud (DeCS). En el caso de que el término necesario no estuviera disponible en el tesauro propio de la base de datos o al usar el portal de búsqueda de Google se emplearon términos libres. Los estudios seleccionados han sido aquellos que hacían referencia a la imagen social de la Enfermería, la invisibilidad de los cuidados o los que abordaban la relación de la imagen de la Enfermería con la prensa, publicados en español o en inglés. No se ha eliminado ningún tipo de diseño de estudio para su inclusión en la revisión, pero se requería acceso al texto completo.

Por otro lado, para recopilar las noticias relacionadas con la Enfermería se ha utilizado la hemeroteca digital de los periódicos El País, El Mundo, Información, La Vanguardia y El Confidencial. Para realizar la búsqueda en los distintos periódicos se ha usado la palabra clave enfermería. Con el fin de realizar el análisis de contenido se siguió el siguiente esquema: lectura del título de la noticia, selección de la misma, lectura en profundidad de las noticias seleccionadas, identificación de aspectos relevantes, identificación de temas recurrentes, clasificación en base a las líneas temáticas detectadas y por último, resumen de resultados en dichas líneas temáticas. Durante esta fase se eliminaron los artículos que hacían referencia a enfermería entendida como estancia física y los que trataban exclusivamente del personal auxiliar de enfermería. El período de revisión de noticias ha sido de 15 años, de tal manera que la noticia más antigua corresponde a noviembre del año 2000 y la más actual a Junio de 2015.

\section{RESULTADOS}

En la figura 1 se muestra un diagrama de flujo que resume el proceso de búsqueda de la revisión bibliográfica y los resultados que han sido hallados.

Con respecto a la segunda fase, se seleccionaron y analizaron un total de 235 noticias relacionadas con la Enfermería. Tras el análisis de contenido emergieron 8 categorías que ayudan a explicar la imagen que se refleja de la Enfermería en la prensa escrita. A continuación, se muestra el sistema categorial obtenido tras el análisis:

\section{- Cuestiones laborales o de mercado de tra-} bajo:

Se localizan numerosas noticias referentes a esta categoría: falta de enfermeros para alcanzar la media europea, efectos de los recortes sobre los pacientes, cómo repercuten estos hechos en la salud de los profesionales de Enfermería que se encuentran "estresados y quemados", búsqueda de trabajo en países extranjeros por dificultad en el mercado laboral español, se denuncian contratos basura, sueldos insuficientes, rebajas salariales, falta de regularización de la figura de la enfermera es- 
colar, agresiones a enfermeros o reclamación puestos específicos para especialidades enfermeras.

\section{- Desconfianza en la Enfermería por parte de otros profesionales de la salud:}

Con titulares como "los médicos advierten de los "graves riesgos" de que los enfermeros receten" o "la receta de los enfermeros abre una gran guerra con los médicos”. La Ley del Medicamento, aprobada en 2009 recoge esta posibilidad, pero la oposición de los médicos hace que se demuestre poca unión entre colectivos lo cual puede provocar que los pacientes duden de los profesionales que les atienden.

\section{- Intrusismo laboral:}

Noticias relacionadas con Doulas que ejercen como matronas sin preparación académica, desvalorizando, así, los cuidados enfermeros.

\section{- Funciones, actividades enfermeras y otros logros:}

Escasas noticias van destinadas a comunicar las funciones de los profesionales de Enfermería. Las noticias encontradas en este aspecto son entrevistas a una enfermera gestora de casos, a tres enfermeros de la unidad de cuidados intensivos, la labor de una matrona en el seguimiento del embarazo, en consultas para dejar de fumar o en proyectos de cooperación. Por otro lado, varias noticias hacen referencia a premios a enfermeros, 4 sobre la realización de jornadas científicas de enfermería y 2 sobre la creación de guías.

\section{- Incorrecta utilización de términos para de-} signar a los profesionales de la Enfermería:

En muchas noticias en el titular se habla de enfermeros y en el texto de auxiliares de enfer- mería. Por otra parte, aún se sigue utilizando el término ATS (titulación desaparecida hace más de 30 años). Este mal uso no beneficia la autonomía por la que se lucha ya que se sobreentiende con él la dependencia a la categoría médica.

\section{- Negligencias y delitos:}

Se encuentran noticias que hacen referencia a errores enfermeros que tienen consecuencias sobre la salud de los pacientes, por ejemplo: "la enfermera que cuidaba de Ryan no tenía la formación adecuada”, participación en los casos de niños robados o participación en la falsificación de documentos. Estos hechos pueden hacer pensar de falta de profesionalidad, formación académica insuficiente y aumentar la desconfianza de la población.

\section{- Presencia en el ámbito erótico-sexual:}

Denuncia a declaraciones de un famoso médico respecto a la Enfermería o cárteles publicitarios. Estereotipo que puede contribuir a vulgarizar el rol del profesional enfermero.

\section{- Sucesos:}

En esta categoría encontramos noticias como la de 2 enfermeros uruguayos que matan al menos a 16 pacientes reconociendo que no se encontraban en situación terminal.

\section{DISCUSIÓN}

En general, la imagen que a través de la prensa se nos transmite es la imagen de alguien con un papel secundario asociado a otra profesión, sin responsabilidad, autonomía o capacidad en la toma de decisiones. Se proyecta una imagen pobre, con poco nivel académico, con oportunidades limitadas en su carrera, poco remunerada y con poca valoración social. Por tanto, la prensa devuelve a la socie- 


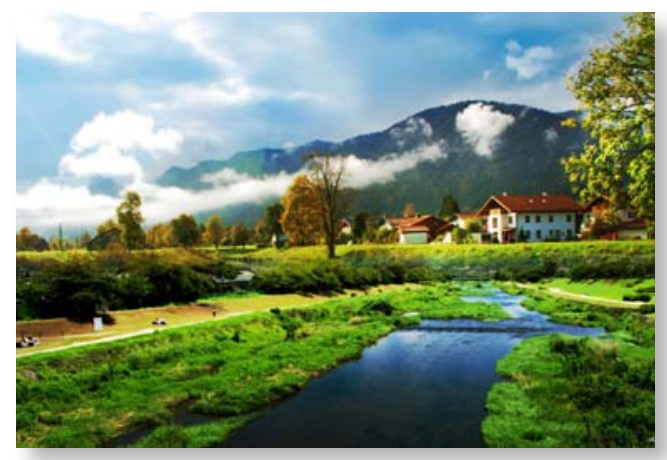

dad una imagen imprecisa y difusa. Asimismo, hay escasos artículos relacionados con el eje principal de la labor enfermera, el cuidado, desde los diferentes campos en los que una enfermera puede desarrollar su trabajo: gestión, docencia, investigación y práctica asistencial, siendo el Información el que mayor tasa de noticias publica de estas características. En cambio, muchas noticias tratan la situación laboral $\mathrm{y}$ las condiciones en los centros de trabajo.

Para transmitir la identidad real de las enfermeras éstas deben participar en el proceso de comunicación a la sociedad de su función y quehacer. Conviene que las instituciones enfermeras cuenten con gabinetes de comunicación bien estructurados, que las enfermeras integren en su práctica profesional el uso de recursos de la comunicación pública, tales como aplicar nuevas tecnologías en la práctica diaria, difundir científica y masivamente su producción intelectual, divulgarla en la sociedad, e informar a los medios de actividades enfermeras susceptibles de convertirse en producto informativo (Calvo, 2011; Avila et al., 2013). Por otro lado, para mejorar la imagen de la Enfermería Ten Hoeve et al. (2013) recomienda que las enfermeras también deben adoptar y desarrollar nuevos roles de liderazgo para ser más visibles. Así como, que la investigación de Enfermería y la práctica basada en la evidencia serán útiles para promover la profesión y resultados de la investigación se podrían utilizar para mejorar el desarrollo de la identidad profesional. Finalmente, como estrategias de visibilidad De la Rosa y Zamora (2012) instan a registrar los cuidados fundamentales de nuestro ejercicio para reconocer la importancia que poseen y comenzar a contabilizarlos dentro del trabajo diario de la profesión.

Como problema metodológico resaltar, que los estudios encontrados en la revisión han sido llevados a cabo en diferentes países $\mathrm{y}$ aunque todos detectan similares problemas en la imagen de la Enfermería hay que ser prudentes a la hora de interpretar los resultados ya que las competencias de la Enfermería dependen del contexto en el que se desarrolla. Debido a los recientes cambios producidos en los estudios universitarios (derivados del plan Bolonia) futuras líneas de investigación para contexto español deberían evaluar y profundizar en el impacto producido en la opinión de la sociedad, de los propios profesionales, de los estudiantes de Enfermería y de los profesionales de comunicación y posteriormente realizar un análisis comparativo.

Claramente es importante mejorar la imagen que el público tiene de las enfermeras. Se insta a realizar iniciativas, como las antes mencionadas, para lograr que los medios enfaticen en las habilidades que poseen, en realidad, las enfermeras, con el objetivo de influenciar en la opinión pública a nivel nacional.

\section{CONCLUSIONES}

A pesar de la importante evolución experimentada podemos afirmar que la imagen de la Enfermería continúa estando muy alejada de la profesión más autónoma, competente y con mayor capacidad de decisión en que se ha convertido. Esta imagen de las enfermeras está en parte muy influenciada por la proyección, que como hemos visto, hacen los medios de comu- 
nicación. El esfuerzo de cambiar la imagen que transmitimos se encuentra en manos de la propia Enfermería. Debemos intentar proyectar públicamente lo mejor de nuestra identidad y lograr así, construir en la mente de la población, para la que trabajamos, una imagen positiva que genere confianza y consecuentemente garantice el máximo aprovechamiento de los recursos que podemos ofrecer y así, lograr una mejora de nuestra posición actual, de la visibilidad de los cuidados enfermeros y de la proyección para el futuro.

\section{REFERENCIAS BIBLIOGRÁFICAS}

- Avila, L.I., Silveira, R.S., Lunardi, V.L., Fernandes, G.F.M., Mancia, J.R., y Silveira, J.T. (2013). Implications of the visibility of profesional nursing practices. Revista Gaúcha de Enfermería, 34 (3), 102-9. Disponible en http://www.scielo.br/scielo.php?pid=S1983$\underline{14472013000300013 \& \text { script }=\text { sci_arttext } \& \text { tlng=en }}$

- Bonill de las Nieves, C. (2014). ¿Cuidados invisibles o profesionales invisibles? Archivos de la Memoria, 11 (1). Disponible en http://www.index-f.com/memoria/11/11200.php

- Calvo Calvo, M.A. (2011). Imagen social de las enfermeras y estrategias de comunicación pública para conseguir una imagen positiva. Index de Enfermería, 20 (3). Disponible: http://scielo.isciii.es/scielo.php?pid=S1132$12962011000200010 \&$ script $=$ sci_arttext

- De la Rosa Eduardo, R., y Zamora Monge, G. (2012). Cuidados invisibles: ¿son suficientemente reconocidos? Index de Enfermería, 21 (4). Disponible en http://scielo.isciii.es/scielo.php?script=sci_arttext\&pid =S1132-12962012000300009

Errasti-Ibarrondo, B., Arantzamendi-Solabarrieta, M., y Canga-Armayor, N. (2012). La imagen social de la enfermería: una profesión a conocer. Anales del sistema sanitario de Navarra, 35 (2), 269-83. Disponible en http://scielo.isciii.es/scielo.php?pid=S113766272012000200009 \&script $=$ sci_arttext

- Fernández-Salazar, S., y Ramos-Morcillo, A.J. (2013). Comunicación, imagen social y visibilidad de los Cuidados de Enfermería. ENE Revista de Enfermería, 7 (1), 1-10. Disponible en http://ene-enfermeria.org/ojs/index. php/ENE/article/viewFile/256/210

- Heirle Valero, C. (2009). La imagen de la enfermera a través de los medios de comunicación de masas: la prensa escrita. Index de Enfermería, 18(2). Disponible en http://scielo.isciii.es/scielo.php?pid=S1132$12962009000200005 \&$ script $=$ sci_arttext

- Huércanos Esparza, I. (2010). El cuidado invisible, una dimensión de la profesión enfermera. Biblioteca Lasca- sas, 6 (1). Disponible en http://www.index-f.com/lascasas/documentos/lc0510.pdf

- Huércanos Esparza, I. (2013). Cuidado invisible: donde los medicamentos no llegan. Index de Enfermería, 22 (1-2). Disponible en http://www.index-f.com/indexenfermeria/v22n1-2/0506.php

- Kalisch, B.J., Begeny, S., y Neumann, S. (2007). The image of the nurse on the Internet. Nursing Outlook, 55 (4), 182-8.

- Kemmer, L.F., y Silva, M.J.P. (2007). La visibilidad del enfermero según la percepción de los profesionales de la comunicación. Revista Latino-Americana de Enfermagem, 15(2). Disponible en http://www.scielo.br/pdf/rlae/ v15n2/es_v15n2a02.pdf

- Marchador Pinillos, B. (2005). Influencia de la prensa escrita en la imagen de la profesión enfermera. Biblioteca Lascasas, 1, 1-33. Disponible en http://www.index-f. com/lascasas/documentos/lc0011.pdf

- Martins Cardoso, R.J., García de Nascimento Graveto, J.M., y Correia Albuquerque Queiroz, A.M. (2014). Visibilidad de la enfermería en la prensa y en la media online. Revista Latino-Americana de Enfermagem, 22 (1), 1-6. Disponible en http://www.scielo.br/pdf/rlae/v22n1/ es_0104-1169-rlae-22-01-00144.pdf

- McAllister, M., Downer, T., Hanson, J., y Oprescu, F. (2014). Transformers: Changing the face of nursing and midwifery in the media. Nurse Education in Practice, 14, 148-53.

- Orkaizagirre Gómara, A. (2013). Visibilizando los cuidados enfermeros a través de la relación de cuidado. Index de Enfermería, 22(3). Disponible en http://www.index-f. com/index-enfermeria/v22n3/1246.php

- Rodríguez Camero, M.L., Rodríguez Camero, N., y Azañón Hernández, R. (2008). La construcción mediática de la Enfermería. Index de Enfermería, 17 (2). Disponible en http://scielo.isciii.es/scielo.php?script=sci_arttext\&pi $\mathrm{d}=\mathrm{s} 1132-12962008000200009$

- Takase, M., Kershaw, E., y Burt, L. (2002). Does Public Image of Nurses Matter? Journal of Professional Nursing, 18 (4), 196-205.

- Ten Hoeve, Y., Jansen, G., y Roodbol, P. (2013). The nursing profession: public image, self-concept and profesional identity. A discussion paper. Journal of Advenced Nursing, 70 (2), 295-309. 